\title{
Dynamical systems, SIMP, bone remodeling and time dependent loads
}

\author{
Anders Klarbring and Bo Torstenfelt
}

\section{Linköping University Post Print}

N.B.: When citing this work, cite the original article.

The original publication is available at www.springerlink.com:

Anders Klarbring and Bo Torstenfelt, Dynamical systems, SIMP, bone remodeling and time dependent loads, 2012, Structural and multidisciplinary optimization (Print), (45), 3, 359-366. http://dx.doi.org/10.1007/s00158-011-0724-x

Copyright: Springer Verlag (Germany) http://www.springerlink.com/

Postprint available at: Linköping University Electronic Press http://urn.kb.se/resolve?urn=urn:nbn:se:liu:diva-77543 


\title{
Dynamical systems, SIMP, bone remodeling and time dependent loads
}

\author{
Anders Klarbring \& Bo Torstenfelt \\ Mechanics \\ Department of Management and Engineering \\ The Institute of Technology \\ Linköping University \\ SE-581 83 Linköping, Sweden \\ E-mail: anders.klarbring@liu.se
}

\begin{abstract}
The dynamical systems approach to sizing and SIMP topology optimization, introduced in a previous paper, is extended to the case of timevarying loads. A general dynamical system, satisfying a Lyaponov-type descent condition, is derived and specialized to a goal function combining stiffness and mass. For a cyclic time-dependent load it is indicated how, in the limit of short cycles compared to the overall time scale, this can be handled by multiple load cases. Numerical examples, both for a convex and a non-convex case, illustrates the theory.
\end{abstract}

\section{Introduction}

In a previous paper, Klarbring and Torstenfelt [11], we introduced a dynamical systems approach to sizing and SIMP $^{1}$ topology optimization. In this approach optimization is viewed as a time dependent evolution process and a central modeling concept is the Lyaponov inequality: the dynamical system should be such that a goal function never increases during an evolution.

In Klarbring and Torstenfelt [11] it was, moreover, shown that an existing model of bone remodeling, see $[5,6,7]$, can be put in one-to-one correspondence with a dynamical system, derived from a SIMP or density model of topology optimization. Thus, the time variable of this dynamical system, initially introduced as an artificial variable, can also be regarded as the real time variable of bone remodeling. Once this is realized (at least) two interesting directions of research emerge and are implemented in this paper: (i) In bone remodeling applications a purely static load is obviously not very common (consider e.g. walking), while in in the optimization context it has so far been the rule. In the present paper we extend our dynamical systems approach of SIMP topology optimization to include time varying loads, strengthening the connection to bone

\footnotetext{
${ }^{1}$ The acronym SIMP stands for Solid isotropic Material with Penalization and is essentially an approach for penalizing intermediate design variable values when optimizing stiffness under a volume constraint (or the reversed). For a detail account we refer to Bendsøe and Sigmund [2] and Christensen and Klarbring [3].
} 
remodeling further. (ii) The loading usually seen in bone remodeling applications is cyclic (again consider walking). Can such cyclic loading be connected to the structural optimization usage of multiple (static) load cases? In the present paper we show that this is indeed the case if the remodeling takes place in a time scale that is sufficiently longer than that of a loading cycle, say months and years compared to seconds.

We like to emphasize that, even though we do not rule out the possibility of using the dynamical systems approach as a vehicle for obtaining more efficient algorithms, this is not the aim this paper. Rather, we like to clarify and strengthen the connection between models of evolutionary problems such as bone remodeling and damage evolution, on the one hand, and structural optimization formulations, on the other. Such a connection is hinted at in several publications, see, e.g., Achtziger et al. [1] and Jang et al. [8], but since the dynamical systems concept has been missing, this has not been achieve to a full extend in our view. These issues were further discussed in our previous paper [11].

In Section 2 we develop a general dynamical system satisfying a Lyaponov inequality. This is done for both constant (in subsection 2.1) and time-varying (in subsection 2.2) external loads. In subsection 2.3 a two-scale view is introduced and it is shown how this coincides with a multiple load case approach under certain conditions. The weighted sum of compliance and mass is introduced as a particular goal function in subsection 2.4, and this is the example for which we also develop the algorithm in Section 3. Numerical calculations are finally reported in subsection 3.2. It is here demonstrated how a two-scale approach approximates the full solution of the cyclic case.

\section{Theory}

We consider a discrete or discretized structure with the following semi-definite stiffness matrix that depends on a vector $\boldsymbol{\rho}=\left(\rho_{1}, \ldots, \rho_{E}\right)^{T}$ :

$$
\boldsymbol{K}(\boldsymbol{\rho})=\sum_{i=1}^{E} \rho_{i}^{q} \boldsymbol{K}_{i},
$$

where $E$ is the number of elements in the structure and $\boldsymbol{K}_{i}$ is an element stiffness matrix. We like to give two different interpretations of the variables $\rho_{i}$ and the exponent $q$. The first interpretation is related to the so-called SIMP formulation of topology optimization. In this case $\boldsymbol{\rho}$ is a vector of design variables that takes values in the interval $[0,1]$, and by taking the exponent $q>1$, interior points of the interval are penalized in an optimization process. The physical interpretation is that $\rho_{i}=0$ corresponds to an element having no stiffness and, thus, being essentially removed from the structure (a hole), while $\rho_{i}=1$ corresponds to an element with full stiffness $\boldsymbol{K}_{i}$. Intermediate values can be viewed as defining an isotropic composite material, see [2]. The second interpretation of $\rho_{i}$ and the exponent $q$ comes from bone remodeling theory, see $[5,6,7]$. Here, $\rho_{i}$ represents the apparent density of the bone and experiments have shown that the stiffness of the bone depends on this density as a power of 2 to 3 , meaning that $q$ takes these values ${ }^{2}$. Clearly such a density has a zero lower bound and an upper bound

\footnotetext{
${ }^{2}$ In the generalized version of bone remodeling theory, presented by Harrigan and Hamilton [6], $q=N / m$, where $N$ is the exponent of the stiffness relation, $m$ is a positive constant and
} 
that is the density of compact bone. Intermediate values represent trabecular (cancellous or spongy) bone.

To incorporate both of these interpretations of (1) we will call $\boldsymbol{\rho}$ a vector of configurational variables and assume that it belongs to the set

$$
K=\prod_{i=1}^{E} K_{i}, \quad K_{i}=\left\{\rho_{i} \mid \underline{\rho} \leq \rho_{i} \leq \bar{\rho}\right\},
$$

where $\underline{\rho}$ and $\bar{\rho}$ are lower and upper bounds on $\rho_{i}$.

The basic equilibrium equation for a linear elastic structure is now

$$
\boldsymbol{F}=\boldsymbol{K}(\boldsymbol{\rho}) \boldsymbol{u},
$$

where $\boldsymbol{u}$ is a vector of nodal displacements and $\boldsymbol{F}$ is the corresponding nodal forces.

\subsection{The case of constant forces}

The design problem generally consists of finding configurational variables such that specific requirements are meet (not necessarily one of optimality). In the present paper we choose to introduce this by stating that a given goal or objective function $f=f(\boldsymbol{u}, \boldsymbol{\rho})$ should never increase during a quasi-static evolution of the system, i.e.,

$$
\frac{d}{d t} f(\boldsymbol{u}, \boldsymbol{\rho}) \leq 0 \quad \text { for all } \boldsymbol{u} \text { and } \boldsymbol{\rho} \in K \text { satisfying } \boldsymbol{F}=\boldsymbol{K}(\boldsymbol{\rho}) \boldsymbol{u},
$$

where $t$ is a time-like variable and $\boldsymbol{u}=\boldsymbol{u}(t)$ and $\boldsymbol{\rho}=\boldsymbol{\rho}(t)$. For biological systems such as bone remodeling this means that we have observed some property, such as increasing stiffness, which seems to be the goal of adaptation. For the topology optimization interpretation, (3) is related to a dynamical systems approach to optimization, see [11]. This means that the optimization problem

$$
\min _{\boldsymbol{u}, \boldsymbol{\rho} \in K} f(\boldsymbol{u}, \boldsymbol{\rho}) \quad \text { subject to } \boldsymbol{F}=\boldsymbol{K}(\boldsymbol{\rho}) \boldsymbol{u}
$$

is solved by defining a dynamical system with the property (3). The equilibrium points of many such dynamical systems coincide with the stationary points of (4), and (3) is the essential condition that is required from a Lyaponov function: if, in addition to this condition, $f$ is radially unbounded and bounded below, the dynamical system is guaranteed to converge to an equilibrium point. Because of this connection we call (4), and similar inequalities, Lyaponov inequalities. Note, however, that the requirement (3) is more general than requiring an optimal point of (4), since there are dynamical systems that satisfy (3) but do not converge to a stationary point of (4).

In the following we will derive a general dynamical system that satisfies (3). To that end, we like to relax the equilibrium constraint of (3). Taking the time derivative of this constraint (for constant $\boldsymbol{F}$ ) we obtain

$$
\sum_{i=1}^{E} \frac{\partial \boldsymbol{K}(\boldsymbol{\rho})}{\partial \rho_{i}} \boldsymbol{u} \dot{\rho}_{i}+\boldsymbol{K}(\boldsymbol{\rho}) \dot{\boldsymbol{u}}=\mathbf{0} .
$$

$\rho_{i}^{1 / m}$ is the apparent density. 
By multiplying this equation by a Lagrangian multiplier vector $\boldsymbol{\lambda}$ and adding to the inequality of (3) we obtain

$$
\left[\nabla_{u} f(\boldsymbol{u}, \boldsymbol{\rho})^{T}+\boldsymbol{\lambda}^{T} \boldsymbol{K}(\boldsymbol{\rho})\right] \dot{\boldsymbol{u}}+\sum_{i=1}^{E}\left[\frac{\partial f(\boldsymbol{u}, \boldsymbol{\rho})}{\partial \rho_{i}}+\boldsymbol{\lambda}^{T} \frac{\partial \boldsymbol{K}(\boldsymbol{\rho})}{\partial \rho_{i}} \boldsymbol{u}\right] \dot{\rho}_{i} \leq 0,
$$

which should be satisfied for all evolutions of $\boldsymbol{u}, \boldsymbol{\lambda}$ and $\boldsymbol{\rho} \in K$. We achieve this requirement by assuming the adjoint system

$$
\nabla_{u} f(\boldsymbol{u}, \boldsymbol{\rho})+\boldsymbol{K}(\boldsymbol{\rho}) \boldsymbol{\lambda}=\mathbf{0},
$$

where we have used the symmetry of $\boldsymbol{K}(\boldsymbol{\rho})$, and the inequality

$$
\sum_{i=1}^{E} R_{i} \dot{\rho}_{i} \geq 0
$$

where

$$
-R_{i} \in \frac{\partial f(\boldsymbol{u}, \boldsymbol{\rho})}{\partial \rho_{i}}+\boldsymbol{\lambda}^{T} \frac{\partial \boldsymbol{K}(\boldsymbol{\rho})}{\partial \rho_{i}} \boldsymbol{u}+\partial I_{K_{i}}\left(\rho_{i}\right),
$$

and $\partial I_{K_{i}}\left(\rho_{i}\right)$ is a subdifferential of the indicator function of the set $K_{i}$. To prove that (6) and (7) imply (5) we use that, see [12]

$$
\begin{aligned}
\partial I_{K_{i}}\left(\rho_{i}\right)=\left\{\underline{\lambda}_{i}+\bar{\lambda}_{i} \mid \underline{\lambda}_{i} \geq 0, \underline{\rho} \leq \rho_{i},\right. & \underline{\lambda}_{i}\left(\underline{\rho}-\rho_{i}\right)=0, \\
& \left.\bar{\lambda}_{i} \geq 0, \rho_{i} \leq \bar{\rho}, \bar{\lambda}_{i}\left(\rho_{i}-\bar{\rho}\right)=0\right\} .
\end{aligned}
$$

It is easily shown, by taking the time derivative of $\underline{\lambda}_{i}\left(\underline{\rho}-\rho_{i}\right)=0$, that $\underline{\lambda}_{i} \dot{\rho}_{i}=0$, and, similarly, that $\bar{\lambda}_{i} \dot{\rho}_{i}=0$. Using these facts and (6), when inserting (8) into (7), we conclude that the inequality (5) is satisfied. This type of proof of satisfaction of (5) is borrowed from a proof of thermodynamic consistency for a system involving a set constraint, first suggested by Strömberg [17].

As a final step in obtaining a system that satisfies the Lyaponov inequality (3) we need to satisfy (7) for all $\dot{\rho}_{i}$. This follows by taking $R_{i}=k_{i} \dot{\rho}_{i}$ for a nonnegative function $k_{i}$, and this function could, e.g., depend on $\boldsymbol{\rho}, \boldsymbol{u}$ and their time derivatives.

The full system of equations describing the evolution of $\boldsymbol{u}$ and $\boldsymbol{\rho}$ (and $\boldsymbol{\lambda}$ ) for a given (fixed) force $\boldsymbol{F}$ now consists of (2), (6) and

$$
0 \in \frac{\partial f(\boldsymbol{u}, \boldsymbol{\rho})}{\partial \rho_{i}}+\boldsymbol{\lambda}^{T} \frac{\partial \boldsymbol{K}(\boldsymbol{\rho})}{\partial \rho_{i}} \boldsymbol{u}+k_{i} \dot{\rho}_{i}+\partial I_{K_{i}}\left(\rho_{i}\right) .
$$

The term $\boldsymbol{\lambda}^{T} \frac{\partial \boldsymbol{K}(\boldsymbol{\rho})}{\partial \rho_{i}} \boldsymbol{u}$ appearing in this equation is known as (the negative of) the mutual energy, see, e.g., Taylor and Bendsøe [19]. Note that the sign of $\dot{\rho}_{i}$ is determined by the relation between this energy and the partial derivative of the goal function and to that end it is interesting to note that if $\boldsymbol{K}(\boldsymbol{\rho})$ is non-singular, so that, by (2), we can view the displacement as a function of the design, i.e., $\boldsymbol{u}=\boldsymbol{u}(\boldsymbol{\rho})$, then

$$
\frac{d f(\boldsymbol{u}(\boldsymbol{\rho}), \boldsymbol{\rho})}{d \rho_{i}}=\frac{\partial f(\boldsymbol{u}, \boldsymbol{\rho})}{\partial \rho_{i}}+\boldsymbol{\lambda}^{T} \frac{\partial \boldsymbol{K}(\boldsymbol{\rho})}{\partial \rho_{i}} \boldsymbol{u} .
$$


Note also that the constraint $\boldsymbol{\rho} \in K$ is included in (8). In fact, when the previous remark holds, (8) is equivalent to the following definition of forces as (generalized) derivative of a potential

$$
-\boldsymbol{R} \in \partial f^{e}(\boldsymbol{\rho}),
$$

where $\boldsymbol{R}=\left(R_{1}, \ldots, R_{E}\right)^{T}$ and $f^{e}(\boldsymbol{\rho})=f(\boldsymbol{u}(\boldsymbol{\rho}), \boldsymbol{\rho})+\sum_{i=1}^{E} I_{K_{i}}\left(\rho_{i}\right)$ is an extended objective function.

\subsection{The case of time dependent loads}

The evolution equation (9) was derived for a fixed force $\boldsymbol{F}$ and a goal function that did not depend explicitly on time. If these assumptions are relaxed, i.e., $f=f(\boldsymbol{u}, \boldsymbol{\rho}, t)$ and $\boldsymbol{F}=\boldsymbol{F}(t)$, we could still end up with a system defined by $(2),(6)$ and (9), granted that we start from the following Lyapunov inequality:

$$
\begin{aligned}
& \frac{d}{d t} f(\boldsymbol{u}, \boldsymbol{\rho}, t) \leq \frac{\partial f(\boldsymbol{u}, \boldsymbol{\rho}, t)}{\partial t}+\nabla_{u} f(\boldsymbol{u}, \boldsymbol{\rho})^{T} \frac{\partial \boldsymbol{u}}{\partial t} \\
& \text { for all } \boldsymbol{u} \text { and } \boldsymbol{\rho} \in K \text { satisfying } \boldsymbol{F}=\boldsymbol{K}(\boldsymbol{\rho}) \boldsymbol{u},
\end{aligned}
$$

where $\partial \boldsymbol{u} / \partial t$ is any function that satisfies $\dot{\boldsymbol{F}}=\boldsymbol{K}(\boldsymbol{\rho})(\partial \boldsymbol{u} / \partial t)$. This makes perfectly good sense since, from a thermodynamical analogy, the right hand side could be seen as the inflow of goal function value, i.e., an increase in such value that is not associated with configurational changes.

\subsection{Two-scale approach $\approx$ multiple load cases}

In the applications of the above theory to bone remodeling there are two very different time scales present: the growth of bone occurs on a scale of month and years while the change of external load occurs on a scale of seconds in a cyclic manner (consider walking). Therefore we will extend the above theory by introducing these two scales. The slow time scale is denoted $t$ and the fast time scale is denoted $\tau$ and these are connected as $t=\tau \varepsilon$, where $\varepsilon$ is the length of a cycle of the external loading $\boldsymbol{F}$, implying that one can consider $\tau$ as dimensionless and belonging to the interval $[0,1)$ during a cycle. As $\varepsilon$ approaches zero we assume that $\boldsymbol{\rho}=\boldsymbol{\rho}(t), \boldsymbol{u}=\boldsymbol{u}(t, \tau)$ and $\boldsymbol{F}=\boldsymbol{F}(t, \tau)$, i.e., while the structural behavior (represented by $\boldsymbol{u}$ and $\boldsymbol{F}$ ) varies both in a quick cyclic manner and in an overall average manner, it is assumed that the growth behavior (represented by $\boldsymbol{\rho}$ ) is so slow that no essential change in the configurational variable occurs in the fast time scale. These assumptions are reminiscent of the multi-scale expansion underlying homogenization theory, which has seen considerable attention in topology optimization, see [2], although in that case one is concerned with variations in space instead of time. Integrating the Lyaponov inequality (3) over one cycle we obtain what is now a new basic design requirement that replaces (3):

$$
\frac{d}{d t} \int_{0}^{1} f(\boldsymbol{u}, \boldsymbol{\rho}) d \tau \leq 0 \quad \text { for all } \boldsymbol{u} \text { and } \boldsymbol{\rho} \in K \text { satisfying } \boldsymbol{F}=\boldsymbol{K}(\boldsymbol{\rho}) \boldsymbol{u} .
$$

Using the above assumptions concerning functional dependencies and taking the force to be independent of $t$, i.e., varying only only in a cyclic manner while the 
mean value is constant, the steps leading to (6) and (7) can be repeated also for the integrated inequality (11) and $R_{i}$ should now be defined as

$$
-R_{i} \in \int_{0}^{1}\left[\frac{\partial f(\boldsymbol{u}, \boldsymbol{\rho})}{\partial \rho_{i}}+\boldsymbol{\lambda}^{T} \frac{\partial \boldsymbol{K}(\boldsymbol{\rho})}{\partial \rho_{i}} \boldsymbol{u}\right] d \tau+\partial I_{K_{i}}\left(\rho_{i}\right),
$$

where $\boldsymbol{\lambda}=\boldsymbol{\lambda}(t, \tau)$. Concerning a time dependent goal function or/and a load depending also on $t$, i.e., when the amplitude of the cycles are varying, as discussed in subsection 2.2, this can also be given a two-scale treatment by using integrals in front of every term in (10).

This two-scale view is akin to the classical treatment of multiple load cases in structural optimization. To make this clear we discretize the integral in (11) as follows:

$$
\int_{0}^{1} f(\boldsymbol{u}, \boldsymbol{\rho}) d \tau \approx \sum_{\ell=1}^{L} \alpha_{\ell} f\left(\boldsymbol{u}\left(\tau_{\ell}, t\right), \boldsymbol{\rho}(\boldsymbol{t})\right),
$$

where $\alpha_{\ell}$ are integration coefficients and $\tau_{\ell} \in[0,1]$ are integration points. The discretized form of the Lyapunov inequality (11) can now be related to the following optimization problem:

$$
\begin{cases}\min _{\boldsymbol{u}_{\ell}, \boldsymbol{\rho} \in K} & \sum_{\ell=1}^{L} \alpha_{\ell} f\left(\boldsymbol{u}_{\ell}, \boldsymbol{\rho}\right) \\ \text { subject to } & \boldsymbol{F}_{\ell}=\boldsymbol{K}(\boldsymbol{\rho}) \boldsymbol{u}_{\ell}, \ell=1, \ldots, L,\end{cases}
$$

where $\boldsymbol{F}_{\ell}$ corresponds to $\boldsymbol{F}\left(\tau_{\ell}\right)$ and $\boldsymbol{u}_{\ell}$ corresponds to $\boldsymbol{u}\left(\tau_{\ell}, t\right)$, where the latter is independent of $t$ when $\boldsymbol{F}$ only depends on $\tau$. Clearly, this is a classical structural optimization problem with multiple load cases.

\subsection{Special goal functions}

In topology optimization the most common components of goal functions are compliance and weight. Compliance is defined as

$$
C=\frac{1}{2} \boldsymbol{F}^{T} \boldsymbol{u}
$$

and will, due to (2), coincide with the strain energy $\Psi$. The weight is a linear function of the configurational variables, i.e.,

$$
W=\sum_{i=1}^{E} a_{i} \rho_{i}
$$

for some constants $a_{i}$. If $\rho_{i}$ is a density as in bone remodeling, $a_{i}$ will be the volume of element $i$. However, we could think of generalizing this so that $W$ represents some general cost of material. Such a cost could be non-linear in $\boldsymbol{\rho}$, see Klarbring et al. [10]. A natural goal function is now

$$
f=C+\mu W,
$$

for some constant $\mu$. In this case

$$
\frac{\partial f(\boldsymbol{u}, \boldsymbol{\rho})}{\partial \rho_{i}}=\mu a_{i}, \quad \nabla_{u} f(\boldsymbol{u}, \boldsymbol{\rho})=\frac{1}{2} \boldsymbol{F} \stackrel{(6)}{\Longrightarrow} \quad \boldsymbol{\lambda}=-\frac{\boldsymbol{u}}{2} .
$$


Using these results, together with the assumption that $\boldsymbol{K}(\boldsymbol{\rho})$ is non-singular for all $\boldsymbol{\rho} \in K$ so that, using (2), $\boldsymbol{u}$ can be seen as a function $\boldsymbol{\rho}$, we find that (9) can be written as

$$
0 \in \mu a_{i}-e_{i}(\boldsymbol{\rho})+k_{i} \dot{\rho}_{i}+\partial I_{K_{i}}\left(\rho_{i}\right)
$$

where we have used the notation

$$
e_{i}(\boldsymbol{\rho})=\frac{1}{2} \boldsymbol{u}^{T} \frac{\partial \boldsymbol{K}(\boldsymbol{\rho})}{\partial \rho_{i}} \boldsymbol{u}
$$

The corresponding formula for the two-scale or multiple load case treatment of section 2.3, after the discretization in (13) is applied, is obtained simply by substituting

$$
\frac{1}{2} \sum_{\ell=1}^{L} \alpha_{\ell} \boldsymbol{u}_{\ell}^{T} \frac{\partial \boldsymbol{K}(\boldsymbol{\rho})}{\partial \rho_{i}} \boldsymbol{u}_{\ell}
$$

for $e_{i}(\boldsymbol{\rho})$ in (15).

As a side remark we mention that another possible combination of weight and compliance was suggested by Strömberg [18], based on an idea of Rozvany $[13,14]$ :

$$
f=C W^{\eta},
$$

where $\eta$ is an adjustable parameter similar to $\mu$ above. For this $f$ we find

$$
\frac{\partial f(\boldsymbol{u}, \boldsymbol{\rho})}{\partial \rho_{i}}=C \eta W^{\eta-1} a_{i}, \quad \nabla_{u} f(\boldsymbol{u}, \boldsymbol{\rho})=\frac{1}{2} \boldsymbol{F} W^{\eta} \stackrel{(6)}{\Longrightarrow} \boldsymbol{\lambda}=-\frac{\boldsymbol{u}}{2} W^{\eta} .
$$

Implying, that (9) now reads

$$
0 \in C \eta W^{\eta-1} a_{i}-W^{\eta} e_{i}(\boldsymbol{\rho})+k_{i} \dot{\rho}_{i}+\partial I_{K_{i}}\left(\rho_{i}\right) .
$$

In order to compare (15) and (18) we note that at equilibrium points $\left(\dot{\rho}_{i}=0\right)$ when $\boldsymbol{\rho} \in K$ is strictly satisfied, (15) gives

$$
\frac{e_{i}(\boldsymbol{\rho})}{a_{i}}=\mu \text {. }
$$

For the same special case (18) gives

$$
\frac{e_{i}(\boldsymbol{\rho})}{a_{i}}=\eta \frac{C}{W} .
$$

Thus, the goal functions (14) and (17) are essentially equivalent in the sense that for a particular solution dependent choice of constants $\mu$ and $\eta$ the same equilibrium points are defined. However, paths leading to such points are obviously different, and from the practical view of computations, one of the two goal functions could turn out to be more useful than the other. However, this is not investigated in the present paper. 


\section{$3 \quad$ Numerical treatment and results}

\subsection{Algorithm}

In the previous paper, Klarbring \& Torstenfelt [11], it was shown that a particular choice of positive function $k_{i}$ in (15), namely

$$
k_{i}=\frac{\mu a_{i}}{\lambda \rho_{i}},
$$

for a positive constant $\lambda$, results in a dynamical system that, when discretized, gives an iteration formula that can be identified with a classical optimality criteria (OC) algorithm, as given, in, e.g., [2]. This is a new interpretation of the OC method and it shows that an iteration sequence produced by this method can be seen as approximating a solution path of a dynamical system. For clarity of presentation we recall below the steps leading from the dynamical system to the OC iteration formula. With the above choice of $k_{i},(15)$ can be written as follows: $\dot{\rho}_{i}=0$ if $\rho_{i}=\bar{\rho}$ and $\partial f / \partial \rho_{i}<0$ or $\rho_{i}=\underline{\rho}$ and $\partial f / \partial \rho_{i}>0$, and otherwise

$$
\mu a_{i} \dot{\rho}_{i}=-\lambda \rho_{i}\left(e_{i}(\boldsymbol{\rho})-\mu a_{i}\right) .
$$

We rewrite this equation as

$$
\frac{d}{d t} \ln \rho_{i}=\lambda\left(\frac{e_{i}(\boldsymbol{\rho})}{\mu a_{i}}-1\right) .
$$

Given a solution $\boldsymbol{\rho}(t)$ at time $t$, we like to calculate an approximation of the solution at time $t+\Delta t$. By an explicit time discretization of the left hand side of equation (19) we calculate test values $\hat{\rho}_{i}(t+\Delta t)$ from

$$
\frac{\ln \hat{\rho}_{i}(t+\Delta t)-\ln \rho_{i}(t)}{\Delta t}=\lambda\left(\frac{e_{i}(\boldsymbol{\rho}(t))}{\mu a_{i}}-1\right),
$$

which we rewrite, using standard formulas for logarithms, as

$$
\left(\frac{\hat{\rho}_{i}(t+\Delta t)}{\rho_{i}(t)}\right)^{\frac{1}{\lambda \Delta t}}=\exp \left(\frac{e_{i}(\boldsymbol{\rho}(t))}{\mu a_{i}}-1\right) \approx \frac{e_{i}(\boldsymbol{\rho}(t))}{\mu a_{i}},
$$

and where the approximation comes from using the first two terms in the Taylor expansion for the exponential. Equation (20) is the OC formula and we can identify the constant $\lambda \Delta t$ as being the so-called damping coefficient. It should, however, be noted that the approximation done in (20) is not essential and in our calculations it is actually not made. In some applications we have even found that this speeds up convergence.

As indicated in section 2.4, the modification that is needed in the formulation and, thus, in the algorithm, to treat the two-scale, or multiple load, case, is to substitute (16) for $e_{i}(\boldsymbol{\rho})$.

As is well known, when the exponent $q$ in (1) is greater than zero, density distributions obtained from the SIMP formulation is prone to oscillations and mesh dependency, see [2] and [3]. Various regularization methods can be used as a remedy for this and were described in the present context in Klarbring \& Torstenfelt [11]. Here we do not repeat this discussion but merely state that all calculations for $q=3$, presented below, uses Sigmunds filter, see [15]. 


\subsection{Comparison of one and two-scale treatment for cyclic loading}

Since the two-scale approach, based on relation (12), corresponds to a limit case when the cycle period goes to zero, it is in general difficult to numerically compare a cyclic one-scale approach to a two-scale one. However, when the cycle is such that $f(\boldsymbol{u}, \boldsymbol{\rho})$ is independent of $\tau$ (for a fixed $\rho$-distribution) we may adjust the loads so that the two goal functions have the same value for a particular $\rho$-distribution. The two formulations then have the potential of converging to the same solution. For a symmetric $\rho$-distribution, geometry and loads as in Figure 1, the objective function (for a fixed $\rho$-distribution) is constant during a load cycle and equal to

$$
f=\frac{1}{2} \boldsymbol{F}^{T} \boldsymbol{u}+\mu W=\frac{1}{2} F \delta+\mu W
$$

Here $\delta$ is the deformation at and in the direction of a load $F$, when only one of the loads are applied.

We like to compare (21) with the objective function for a two-scale approach of the same problem description. We then need to evaluate

$$
f_{T S}=\int_{0}^{1} f(\boldsymbol{u}, \boldsymbol{\rho}) d \tau=\frac{1}{2} \int_{0}^{1} \boldsymbol{F}^{T} \boldsymbol{u} d \tau+\mu W
$$

for the load history shown in Figure 1. The integral of the last expression becomes

$$
\frac{1}{2}\left(\frac{1}{2} F_{1} \delta_{1}+\frac{1}{2} F_{2} \delta_{2}\right)=\frac{1}{2} F \delta,
$$

where, in the last equality, we have assumed $F=F_{1}=F_{2}$, so that the corresponding displacements $\delta_{1}$ and $\delta_{2}$ are such that $\delta=\delta_{1}=\delta_{2}$. Thus, this assumption for the loads gives equal values to the objective functions, which was our aim.

\subsection{Numerical results}

We solve the problem in Figure 1 for three different settings. Our aim is mainly to contrast a one-scale treatment to a two-scale one, but for comparison we have also solved the problem when both loads shown in Figure 1 act simultaneously as one single load case. We have used two different values for the constant $q$ of equation (2), which in the introduction was indicated to be a penalty parameter in the SIMP method, while in the bone remodeling interpretation it is a parameter in the relation between apparent density and material stiffness. It is known, see Stolpe \& Svanberg [16], that the goal function is convex for $q \leq 1$ and non-convex for other values.

In Figure 2 we show the solutions for the convex case $(q=1)$. The number of four-node elements with element-wise constant $\rho$-distribution in the 1 by 1 square is 14400 in all solutions. We take $\bar{\rho}=1$ and $\rho$ equal to a small value. The initial $\rho$-distribution is constant over the whole design domain and such that $\rho_{i}=0.3$. The constant $\mu$ in the goal function (14) is $0.237181 e-7$ which is a value

found by first solving a classical stiffness optimization problem with a constraint 

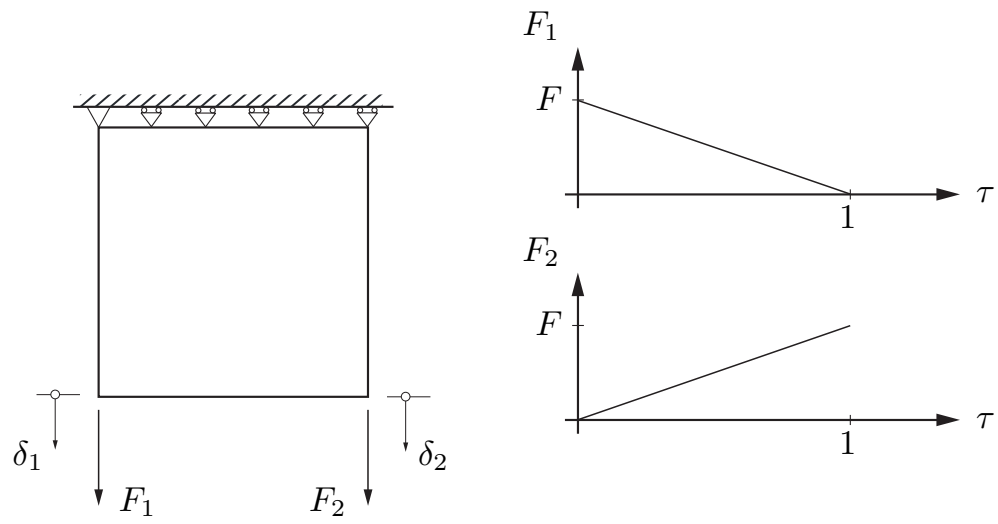

Figure 1: The problem definition. In the right figure it is shown how the loads vary during a loading cycle.
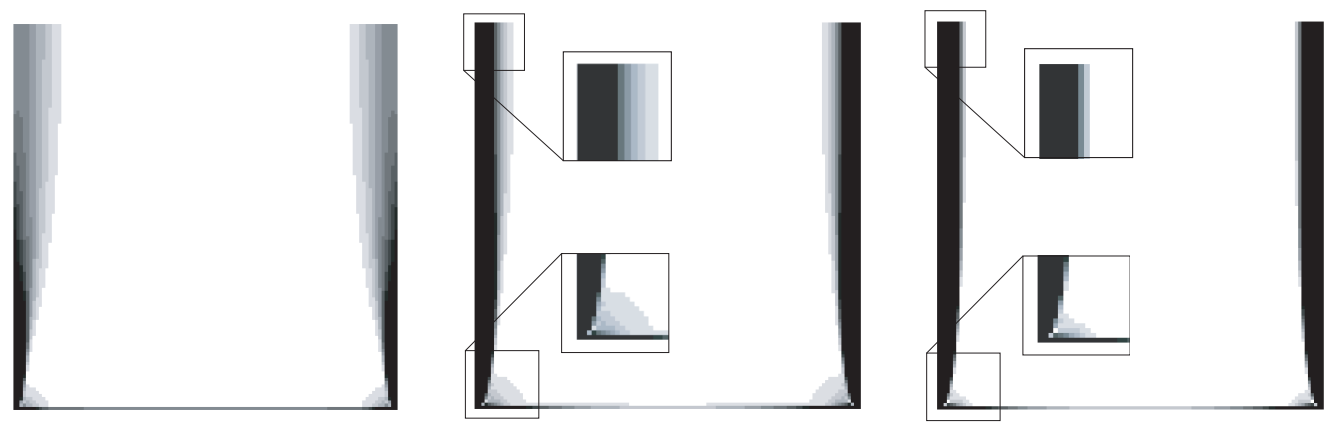

Figure 2: Both loads treated as one simultaneous load case (left), the loads treated as two separate load cases, i.e., a two-scale approach, (middle), and the solution for an oscillating load (right). In all cases $q=1$.

on total volume. The distributions shown in Figure 2 are the final steady-state solutions. The left hand one, where the loads are treated as one single load case, is clearly different from the other two, showing a larger gray region. There is also a difference, but less marked, between the other two cases, clearly indicated in the blow-ups. The middle solution is for the two-scale treatment, which is the same as using two separate load cases. In the right hand solution we use a onescale approach where we resolve each individual cycle. Each cycle is discretized into two time steps, so 2500 cycles are treated since each time step can be seen as one iteration, see Table 1. In the two-scale approach it is possible to use much larger time steps. Since the damping factor translates into time increments as $\lambda \Delta t$, the number of iterations indicated in Table 1 are such that the two-scale solution is integrated only up to 0.6 times the final time of the one-scale solution. However, changes in density distributions between two increments were down to machine precision in both cases. The small but market differences between the two right hand side solutions are attributed to a finite cycle length $\varepsilon$ in the 
one-scale case. Note that in all three solutions, shown in Figure 2, there is a thin

\begin{tabular}{|l|c|c|c|c|}
\hline \hline & \multicolumn{2}{|c|}{$q=1$} & \multicolumn{2}{c|}{$q=3$} \\
\cline { 2 - 5 } & two-scale & one-scale & two-scale & one-scale \\
\hline goal function value & $0.320086 \mathrm{e}-8$ & $0.320421 \mathrm{e}-8$ & $0.397317 \mathrm{e}-8$ & $0.400148 \mathrm{e}-8$ \\
\hline number of iterations & 30 & 5000 & 105 & 5000 \\
\hline damping factor & 0.5 & 0.005 & 0.5 & 0.005 \\
\hline \hline
\end{tabular}

Table 1: Comparison of final goal function values and number of iterations.

horizontal bar at the lower boundary. Horizontal forces in this bar are balanced by the integrated horizontal force in the large gray area (shown almost white in Figure 2). The reason for the presence of such a bar is the sliding boundary conditions at the upper boundary in Figure 1, see the discussion in connection with the non-convex problem below.
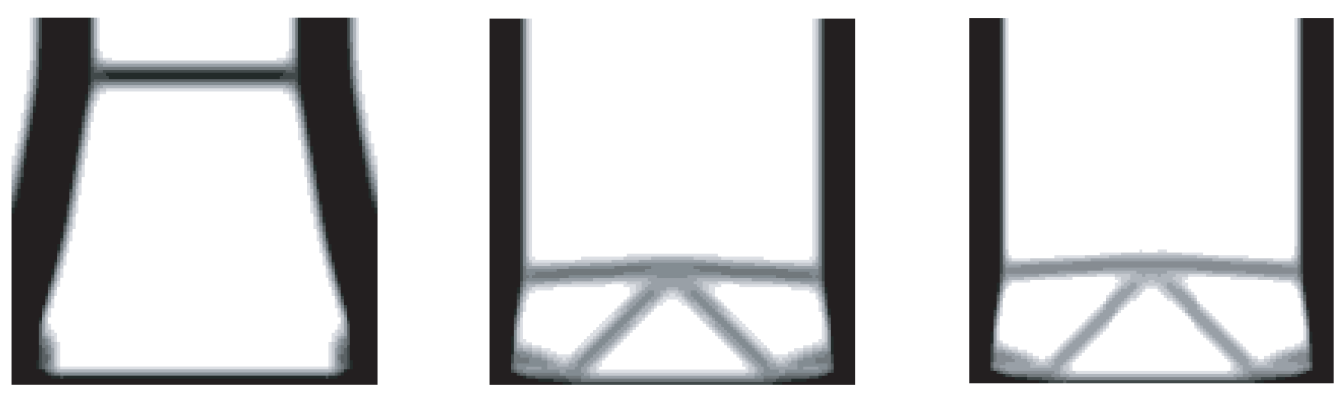

Figure 3: Both loads treated as one simultaneous load case (left), the loads treated as two separate load cases, i.e., a two-scale approach, (middle), and the solution for an oscillating load (right). In all cases $q=3$.

The non-convex case, i.e., $q=3$, is shown in Figure 3 and in the right hand side of Table 1. The number of elements and other data are the same as in the convex case and we use a filter radius that is 0.024 times the side length of the square in Figure 1. The general trends in qualitative differences between the three solutions are the same as in the convex case, but possibly more marked.

We like to comment on the vertical structure present in all three solutions of Figure 3. As indicated above, the reason for this structure is the slinging boundary conditions at the top boundary of the design domain. To understand this fact one may first consider that for a low amount of material used, the optimum structure would consist simply of two vertical columns connecting the forces to the support. When the amount of material exceeds that needed for building these columns (this amount is determined by the upper bound $\bar{\rho}$ and the small area over which the forces are applied), then a slanted structure needs to be added, but such a structure is not effective unless supported horizontally. To further enhance the understanding of this one may think of discrete truss: due to the sliding boundary, a bar that extends non-vertically from the load to the support will be stress free unless somehow supported horizontally. 


\section{Discussion}

The dynamical systems approach to structural adaptation and optimization, introduced in [11] and further developed in this paper, can be viewed in complementary ways. On the one hand, it gives a mathematically well-defined evolutionary approach towards optimal (man-made) structures, complementing the more algorithmic view seen in, e.g., ESO-methods [20]. On the other hand, it describes adaptation that occurs in some natural phenomena such as bone remodeling [6] and damage evolution [1]. The present paper contributes to both of these views by showing how the classical multiple load case situation fits in an evolutionary view. The numerical examples indicate that a two-scale multiple load case treatment approximates a full one-scale cyclic treatment, while being significantly less computationally expensive.

As a final remark it can be pointed out that the models found in $[5,6,7]$, which are here given a new interpretation and are extended to cyclic loading, should be regarded as phenomenological first approximations to the complex processes of bone growth and remodeling. Obviously they do not take into account transport phenomena and bio-chemical processes. However, such developments are possible: in [9] we indicated how the present dynamical systems approach can be extended to involve inflow of "bio-chemical" energy, or "nutrient", that stimulates the growth process. In a forthcoming publication we will also show how the so-called lazy zone concept, see e.g. [4], fits naturally into the present frame.

\section{References}

[1] W. Achtziger, M.P. Bendsøe and J.E. Taylor, Bounds on the effect of progressive structural degradation, Journal of the Mechanics and Physics of Solids 46(6) (1998) 1055-1087.

[2] M.P. Bendsøe and O. Sigmund, Topology optimization: theory, methods and applications, Springer 2003.

[3] P.W. Christensen and A. Klarbring, An Introduction to Structural Optimization, Springer 2009.

[4] J.W.C. Dunlop, M.A. Hartmann, Y.J.Bréchet, P. Fratzl, R. Weinkamer, New suggestions for the mechanical control of bone remodeling, Calcified Tissue International 85 (2009) 45-54.

[5] T.P. Harrigan and J.J. Hamilton, Optimality conditions for finite element simulation of adaptive bone remodeling, International Journal of Solids and Structures 29(23) (1992) 2897-2906.

[6] T.P. Harrigan and J.J. Hamilton, Necessary and sufficient conditions for global stability and uniqueness in finite element simulations of adaptive bone remodeling, International Journal of Solids and Structures 31(1) (1994) 97107.

[7] T.P. Harrigan and J.J. Hamilton, Bone remodeling and structural optimization, Journal of Biomechanics 27(3) (1994) 323-328. 
[8] Gwun Jang, Yong Kim and Byung Man Kwak, Analogy of strain energy density based bone-remodeling algorithm and structural optimization, Journal of Biomechanical Engineering 131 (2009) 011012.

[9] A. Klarbring, Topology optimization, dynamical systems, thermodynamics and growth. In: L. Damkilde, L. Andersen, A.S. Kristensen and E. Lund (Eds.) Proceedings of the Twenty Second Nordic Seminar on Computational Mechanics, Aalborg (2009) 337-344.

[10] A. Klarbring, J. Petersson, B. Torstenfelt and M. Karlsson, Topology optimization of flow networks, Computer Methods in Applied Mechanics and Engineering 192 (2003) 3909-3932.

[11] A. Klarbring and B. Torstenfelt, Dynamical systems and topology optimization, Structural and Multidisciplinary Optimization 42(2) (2010) 179-192.

[12] R.T. Rockafellar, Convex Analysis, Princton University Press 1972.

[13] G.I.N. Rozvany, O.M. Querin, Z. Gaspar and V. Pomezanski, Extended optimality in topology design, Structural and Multidisciplinary Optimization 24 (2002) 257-261.

[14] G.I.N. Rozvany, O.M. Querin, Z. Gaspar and V. Pomezanski, Erratum for the Brief Note Extended optimality in topology design by G.I.N. Rozvany, O.M. Querin, Z. Gaspar, V. Pomezanski, (SMO24, 257261, 2002), Structural and Multidisciplinary Optimization 30 (2005) 504.

[15] O. Sigmund, Morphology-based black and white filters for topology optimization, Structural and Multidisciplinary Optimization 33 (2007) 401-424.

[16] M. Stolpe and K. Svanberg, An alternative interpolation scheme for minimum compliance topology optimization, Structural and Multidisciplinary Optimization 22(2) (2001) 116-124.

[17] N. Strömberg, An augmented Lagrangian method for fretting problems, European Journal of Mechanics, A/Solids 16 (1997) 573-593.

[18] N. Strömberg, Topology optimization of structures with manufacturing and unilateral contact constraints by minimizing an adjustable compliance-volume product, Structural and Multidisciplinary Optimization, to appear

[19] J.E. Taylor and M.P. Bendsøe, A mutal energy formulation for optimal structural design, Structural and Multidisciplinary Optimization 22 (2001)

[20] Y.M. Xie and G.P. Steven, Evolutionary Structural Optimization, Springer 1997.

95-101. 